\title{
Association of noninvasive respiratory support with mortality and intubation rates in acute respiratory failure: a systematic review and network meta-analysis
}

\author{
Hideto Yasuda ${ }^{1,2^{*}}$ (D), Hiromu Okano ${ }^{3}$, Takuya Mayumi ${ }^{4}$, Masaki Nakane ${ }^{5}$ and Nobuaki Shime ${ }^{6}$
}

\begin{abstract}
Background: Noninvasive respiratory support devices may reduce the tracheal intubation rate compared with conventional oxygen therapy (COT). To date, few studies have compared high-flow nasal cannula (HFNC) use with noninvasive positive-pressure ventilation (NPPV). We conducted a network meta-analysis to compare the effectiveness of three respiratory support devices in patients with acute respiratory failure.

Methods: The Cochrane Central Register of Controlled Trials, MEDLINE, EMBASE, and Ichushi databases were searched. Studies including adults aged $\geq 16$ years with acute hypoxic respiratory failure and randomizedcontrolled trials that compared two different oxygenation devices (COT, NPPV, or HFNC) before tracheal intubation were included. A frequentist-based approach with a multivariate random-effects meta-analysis was used. The network meta-analysis was performed using the GRADE Working Group approach. The outcomes were short-term mortality and intubation rate.

Results: Among 5507 records, 27 studies (4618 patients) were included. The main cause of acute hypoxic respiratory failure was pneumonia. Compared with COT, NPPV and HFNC use tended to reduce mortality (relative risk, 0.88 and 0.93 , respectively; $95 \%$ confidence intervals, $0.76-1.01$ and $0.80-1.08$, respectively; both low certainty) and lower the risk of endotracheal intubation (0.81 and 0.78; 0.72-0.91 and 0.68-0.89, respectively; both low certainty); however, short-term mortality or intubation rates did not differ (0.94 and 1.04, respectively; $0.78-1.15$ and 0.88-1.22, respectively; both low certainty) between NPPV and HFNC use.
\end{abstract}

Conclusion: NPPV and HFNC use are associated with a decreased risk of endotracheal intubation; however, there are no significant differences in short-term mortality.

Trial registration: PROSPERO (registration number: CRD42020139105, 01/21/2020)

Keywords: Acute hypoxic respiratory failure, Conventional oxygen therapy, Noninvasive ventilation, High-flow nasal cannula, Systematic review, Meta-analysis, Network meta-analysis

\footnotetext{
* Correspondence: yasudahideto@me.com

'Department of Emergency and Critical Care Medicine, Jichi Medical University Saitama Medical Center, 1-847, Amanuma-cho, Oomiya-ku, Saitama-shi, Saitama 330-8503, Japan

${ }^{2}$ Department of Clinical Research Education and Training Unit, Keio University Hospital Clinical and Translational Research Center (CTR), 35, Shinanomachi, Shinjuku-ku, Tokyo 160-8582, Japan

Full list of author information is available at the end of the article
}

C C The Author(s). 2021 Open Access This article is licensed under a Creative Commons Attribution 4.0 International License, which permits use, sharing, adaptation, distribution and reproduction in any medium or format, as long as you give appropriate credit to the original author(s) and the source, provide a link to the Creative Commons licence, and indicate if changes were made. The images or other third party material in this article are included in the article's Creative Commons licence, unless indicated otherwise in a credit line to the material. If material is not included in the article's Creative Commons licence and your intended use is not permitted by statutory regulation or exceeds the permitted use, you will need to obtain permission directly from the copyright holder. To view a copy of this licence, visit http://creativecommons.org/licenses/by/4.0/ The Creative Commons Public Domain Dedication waiver (http://creativecommons.org/publicdomain/zero/1.0/) applies to the data made available in this article, unless otherwise stated in a credit line to the data. 


\section{Background}

Acute respiratory failure (ARF) is prevalent among critically ill patients and is a common cause of intensive care unit (ICU) mortality [1, 2]. Approximately $60 \%$ of patients with ARF require invasive mechanical ventilation (IMV) [3], which is associated with adverse events, including ventilator-induced lung injury (VILI) and ventilator-associated pneumonia (VAP) $[4,5]$. Patients with ARF on IMV have high hospital mortality rates of up to $30 \%$ [4]. Initial respiratory support, including conventional oxygen therapy (COT; e.g., nasal cannulas and facemasks), noninvasive positive-pressure ventilation (NPPV), and high-flow nasal cannula (HFNC) use, are important treatments to prevent tracheal intubation and reduce mortality among patients with hypoxic respiratory failure [6-10].

There is a widespread application of NPPV in patients with ARF before tracheal intubation and IMV [6-8] which decreases the need for IMV rather than the use of COT [11, 12]. NPPV potentially increases the risk of complications, including aspiration pneumonia, facial skin breakdown, eye irritation, interface intolerance, and patient discomfort from the inability to communicate or eat during therapy $[13,14]$, which limits NPPV application in the clinical setting. HFNC can deliver highconcentration humidified oxygen via nasal cannulas without NPPV-related complications and is increasingly used in critically ill adult patients despite contradictory results from several clinical trials $[9,10]$. However, there is a paucity of evidence on pre-IMV HFNC use in patients with ARF.

Systematic reviews and meta-analyses that compared two of the three respiratory support devices (COT, NPPV, and HFNC) [15-22] showed that HFNC use reduced the tracheal intubation rate compared with COT, albeit without significance between-group differences when compared with NPPV. There was no intergroup difference in mortality between the use of any two of the three respiratory support devices. Several studies in those systematic reviews compared HFNC use with COT and NPPV with COT, although a few studies have compared HFNC use with NPPV. Small sample sizes possibly affected the results of the abovementioned systematic reviews. To overcome these limitations, we performed a systematic review and network meta-analysis (NMA) to compare the effectiveness of three supplemental respiratory support devices in studies that compared at least two of the three techniques (COT, NPPV, and HFNC use) in patients with ARF.

\section{Methods}

\section{Protocol and registration}

This systematic review was designed according to the Preferred Reporting Items for Systematic review and
Meta-Analyses extension statement for reviews incorporating network meta-analyses (details in e-Table 1 in Additional file 1) [23], and the protocol is registered with PROSPERO (CRD42020139105).

\section{Eligibility criteria \\ Type of studies}

We included all randomized-controlled trials (RCTs) reported in English and Japanese regardless of publication status (published, unpublished, and academic abstracts). Randomized crossover, cluster-randomized, and quasiexperimental trials were excluded.

\section{Type of participants}

This review included adults (age $\geq 16$ years) with acute hypoxic respiratory failure, defined by any of the following criteria: ratio of arterial oxygen partial pressure to fractional inspired oxygen (P/F ratio) $<40.00 \mathrm{kPa} ; \mathrm{SaO}_{2}$ or $\mathrm{SpO}_{2}<94 \%$ on room air or $>95 \%$ with $>6 \mathrm{~L} / \mathrm{min}$; and $\mathrm{PaO}_{2}<8.00 \mathrm{kPa}$ with room air or $<10.67 \mathrm{kPa}$ with $\mathrm{O}_{2}$. This meta-analysis excluded studies in which more than half of the patients had post-extubation respiratory failure, acute exacerbation of chronic obstructive pulmonary disease (COPD), acute exacerbation of asthma, hypercapnia (> $6.00 \mathrm{kPa}$ ), tracheostomy, post-surgical status, trauma, and do-not-resuscitate orders. The exclusion criteria were limited to factors that were judged clinically appropriate for exclusion by the participating clinicians.

\section{Types of interventions and comparators}

We included RCTs comparing two of the following three methods before tracheal intubation:

1. COT: Low-flow nasal cannula, face mask, and venturi mask (with no limit on the flow rate).

2. NPPV: The type of mask and mode, duration of ventilation, and methods of weaning were not limited.

3. HFNC: The flow rate and $\mathrm{F}_{1} \mathrm{O}_{2}$ were not limited.

\section{Type of outcomes}

The outcome measures included a primary outcome of short-term mortality at the end of the follow-up period (<90 days), ICU discharge, and hospital discharge. The secondary outcome was the rate of intubation during ICU stay.

\section{Information sources}

We searched for eligible trials in the following databases: The Cochrane Central Register of Controlled Trials (CENTRAL); MEDLINE via PubMed; EMBASE; and Ichushi, a database of Japanese research papers. Additionally, we searched for ongoing trials in The World 
Health Organization International Clinical Trials Platform Search Portal. For cases with unknown data, the authors were contacted.

\section{Search}

We used the search terms "ARDS", "adult respiratory distress syndrome", "respiratory failure", or "acute lung injury" AND "non-invasive ventilation", "NPPV", "oxygen therapy", "HFNC", or "high-flow therapy" in searches performed in December 2020 (details in eTable 2 in Additional file 1).

\section{Study selection}

Two of the three physicians (TM, HO, and HY) screened the title and abstract or the full text at the first and second screenings, respectively, for relevant studies and independently extracted data from the included studies into standardized data forms. Disagreements, if any, were resolved by discussion with one of three physicians who did not screen that particular study; original authors were contacted for clarification as required. For abstract-only studies that could not be evaluated for eligibility based on our review criteria, we attempted to contact the authors. Discrepancies between two reviewers were resolved by mutual discussion or discussion with a third reviewer as needed.

\section{Data collection process and data items}

After identifying studies in the second screening, data were extracted from each study by the reviewers (TM, HO, and HY) using two tools: the Cochrane Data Collection Form (RCTs only) [24] and Review Manager (RevMan) software V.5.3.5 (Cochrane Collaboration) [25]. We extracted the following study characteristics:

(1) Methods: study design, total study duration, number and locations of study centers, study setting, withdrawals, and date of study initiation

(2) Participants: number, mean age, age range, sex, severity of condition, diagnostic criteria, and inclusion/exclusion criteria

(3) Interventions: treatment approaches and comparison methods

(4) Outcomes: primary and secondary outcomes that were specified and collected, and the timepoints reported

\section{Risk of bias within individual studies}

The risk of bias of primary outcomes in the included studies was independently assessed by two of the three authors (TM and HO) using the Cochrane Risk of Bias tool 1.0 (Cochrane Collaboration) $[26,27]$ in seven domains: (a) random sequence generation, (b) allocation concealment, (c) blinding of participants and personnel, (d) blinding of outcome assessors, (e) incomplete outcome data, (f) selective outcome reporting, and (g) other sources of bias. The risk of each bias was graded as low, unclear, or high. Discrepancies between the two reviewers were resolved through discussion among themselves or with a third reviewer as necessary.

\section{Statistical analyses \\ Direct comparison meta-analysis}

A pairwise meta-analysis was performed using RevMan 5.3 (RevMan 2014). Forest plots were used for metaanalysis, and the effect size was expressed as relative risk (RR) with the 95\% confidence interval (CI) for categorical data and as weighted mean differences with the 95\% $\mathrm{CI}$ for continuous data. Outcome measures were pooled using a random effect model for study-specific effects in measures. For all analyses, a two-sided $p$ value $<0.05$ was considered statistically significant.

Study heterogeneity between trials for each outcome was assessed by visually inspecting forest plots and with an $I^{2}$ statistic to quantify inconsistency [28] (RevMan; $I^{2}$ $=0-40,30-60,50-90$, and $75-100 \%$ indicated minimal, moderate, substantial, and considerable heterogeneity, respectively). When heterogeneity was identified $\left(I^{2}>\right.$ $50 \%$ ), we investigated the reason and quantified it using the Chi-square test.

We planned to use a funnel plot, Begg's adjusted rank correlation test, and Egger's regression asymmetry test to investigate publication bias if $\geq 10$ studies were available (RevMan) [29]. As < 10 studies were included, we did not test for funnel plot asymmetry.

\section{Network comparison meta-analysis}

Data synthesis A network plot was constructed to determine the number of studies and patients included in this meta-analysis. An NMA using netmeta 0.9-5 Rpackage (version 3.5.1) was performed via a frequentistbased approach with multivariate random-effects metaanalysis, and effect size was expressed as the RR (95\% CI). Covariance between two estimates from the same study shows the variance of data in the shared arm, as calculated in a multivariable meta-analysis performed using the GRADE Working Group Approach for an NMA [30, 31].

Transitivity The transitivity assumption underlying the NMA was evaluated by comparing the distribution of clinical and methodological variables that could act as effect modifiers across treatment comparisons.

Ranking Ranking plots (rankograms) were constructed based on the probability that a given treatment had the highest event rate for each outcome. The surface under 
the cumulative ranking curve (SUCRA), which is a simple transformation of the mean rank, was used to determine treatment hierarchy [32] and was constructed using standard software (Stata 15.0, Stata, TX, USA).

Risk of bias across studies The assessment of the risk of bias across studies followed considerations of pairwise meta-analysis, and conditions associated with "suspected" and "undetected" across-study bias were determined by the presence of publication bias on a direct comparison.

Indirectness We evaluated the indirectness, classified as "no", "some", or "major" concern, of each study included in the NMA based on its relevance to the research question, including the study population, interventions, outcomes, and study setting. The study-level judgments could be combined with the percentage contribution matrix.

Imprecision The approach to imprecision involved comparing the range of treatment effects included in the 95\% CI with the range of equivalence. We assessed the heterogeneity of treatment effects for a clinically important risk ratio of $<0.8$ or $>1.25$ in the CIs.

Heterogeneity To assess the level of heterogeneity, we compared the posterior and predictive distributions of the estimated heterogeneity variance [33]. Concordance between assessments based on CIs and prediction of intervals, both of which do not capture heterogeneity, were used to assess the importance of heterogeneity of treatment effects for a clinically important risk ratio of $<0.8$ or $>1.25$ in prediction intervals.
Assessment of inconsistency The inconsistency of the network model was estimated using inconsistency factors and their uncertainties. We statistically evaluated consistency using the design-by-treatment interaction test [34]. For inconsistency, $p$ values less than 0.05 , between 0.05 and 0.10 , and otherwise were classified as "Major concerns", "Some concerns", and "No concerns", respectively.

\section{Additional analyses}

If there were sufficient data, we conducted a subgroup analysis of the severity of respiratory failure $(\mathrm{P} / \mathrm{F}<200)$ and the cause of respiratory failure (immunocompromised patients, excluding congestive heart failure (CHF)/acute exacerbation of COPD patients) to investigate the impact of risk of bias and assess the heterogeneity in participants in each study.

\section{Results}

\section{Study selection}

The comprehensive search yielded 5507 records (e-Fig 1 in Additional file 1), of which 27 studies were included in this NMA [9, 35-60]. These 27 studies included two three-group studies that directly compared NPPV with HFNC use and COT. The final analysis included 19, 7, and 5 studies that compared NPPV with COT, HFNC use with COT, and HFNC use with NPPV, respectively. The network structures of each outcome are shown in Fig. 1a and b.

\section{Study characteristics}

The protocols and characteristics of each study included in the final dataset of the meta-analysis are summarized in Table 1 . The quantitative analysis included 4618 patients. The main cause of acute

\section{(a) Short-term mortality}

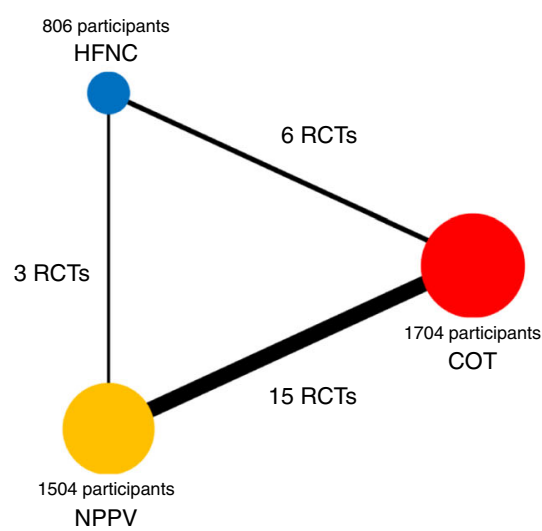

(b) Intubation

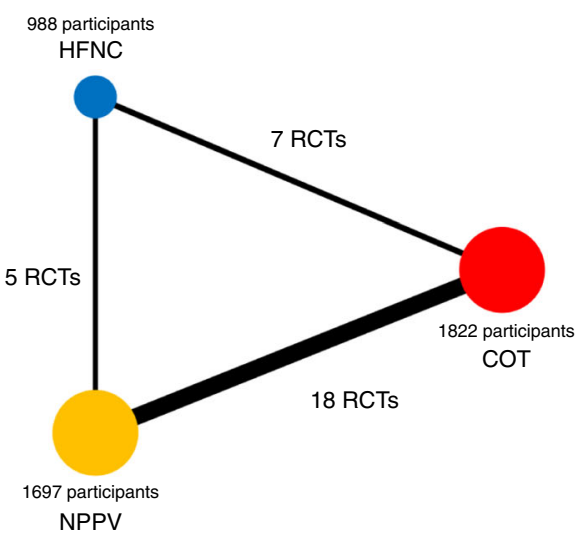

Fig. 1 Network plots for the association of noninvasive oxygenation strategies with short-term mortality and intubation: a short-term mortality b Intubation 
Table 1 Study populations, protocols, and characteristics

\begin{tabular}{|c|c|c|c|c|c|c|c|}
\hline \multirow{2}{*}{$\begin{array}{l}\text { Author, } \\
\text { year }\end{array}$} & \multirow{2}{*}{$\begin{array}{l}\text { Sample } \\
\text { size } \\
n\end{array}$} & \multicolumn{3}{|l|}{ Protocols } & \multicolumn{3}{|l|}{ Baseline characteristics } \\
\hline & & $\begin{array}{l}\text { Intervention } \\
\text { setting }\end{array}$ & $\begin{array}{l}\text { Control } \\
\text { setting }\end{array}$ & Outcomes & $\begin{array}{l}\text { The main causes of acute respiratory } \\
\text { failure }\end{array}$ & Age, years & $\mathrm{PaO}_{2}: \mathrm{F}_{1} \mathrm{O}_{2}$ \\
\hline $\begin{array}{l}\text { Bersten } \\
1991[51]\end{array}$ & 40 & NPPV & COT & $\begin{array}{l}\text { 1. Mortality (in-hospital) } \\
\text { 2. Intubation }\end{array}$ & $\begin{array}{l}\text { CPE } \\
\text { (CHF [56.4\%]) }\end{array}$ & $\begin{array}{l}\text { NPPV: } 76 \\
\text { (6) } \\
\text { COT: } 75 \text { (6) }\end{array}$ & $\begin{array}{l}\text { NPPV: } 138 \\
\text { (32) } \\
\text { COT: } 136 \text { (44) }\end{array}$ \\
\hline $\begin{array}{l}\text { Wysocki } \\
1995[36]\end{array}$ & 41 & NPPV & COT & $\begin{array}{l}\text { 1. Mortality (in-ICU) } \\
\text { 2.Intubation }\end{array}$ & $\begin{array}{l}\text { AHRF } \\
\text { (CHF [30\%]) }\end{array}$ & $\begin{array}{l}\text { NPPV: } 64 \\
(18) \\
\text { COT: } 62 \\
(11)\end{array}$ & $\begin{array}{l}\text { NPPV: } 191 \\
\text { (94) } \\
\text { COT: } 170(77)\end{array}$ \\
\hline $\begin{array}{l}\text { Antonelli } \\
2000[49]\end{array}$ & 40 & NPPV & COT & $\begin{array}{l}\text { 1. Mortality (in-ICU) } \\
\text { 2. Intubation }\end{array}$ & $\begin{array}{l}\text { ARDS } \\
\text { (patients who underwent solid organ } \\
\text { transplantation [100\%]) }\end{array}$ & $\begin{array}{l}\text { NPPV: } 45 \\
\text { (19) } \\
\text { COT: } 44 \\
(10)\end{array}$ & $\begin{array}{l}\text { NPPV: } 142 \\
\text { (29) } \\
\text { COT: } 149(22)\end{array}$ \\
\hline $\begin{array}{l}\text { Delclaux } \\
2000[56]\end{array}$ & 123 & NPPV & COT & $\begin{array}{l}\text { 1. Mortality (in-ICU, in- } \\
\text { hospital) } \\
\text { 2. Intubation }\end{array}$ & $\begin{array}{l}\text { CPE } \\
\text { (infection [49.6\%]) }\end{array}$ & $\begin{array}{l}\text { NPPV: } 56 \\
{[19-85]^{a}} \\
\text { COT: } 60 \\
{[18-88]^{a}}\end{array}$ & $\begin{array}{l}\text { NPPV: } 140 \\
{[59-288]^{a}} \\
\text { COT: } 148 \\
{[62-283]^{a}}\end{array}$ \\
\hline $\begin{array}{l}\text { Masip } \\
2000 \text { [39] }\end{array}$ & 37 & NPPV & COT & 1. Intubation & $\begin{array}{l}\text { CPE } \\
\text { (myocardial infarction [29.7\%]) }\end{array}$ & $\begin{array}{l}\text { NPPV: } 75 \\
(11) \\
\text { COT: } 79 \text { (5) }\end{array}$ & NA \\
\hline $\begin{array}{l}\text { Hilbert } \\
2001[46]\end{array}$ & 52 & NPPV & COT & $\begin{array}{l}\text { 1. Mortality (in-ICU, in- } \\
\text { hospital) } \\
\text { 2. Intubation }\end{array}$ & $\begin{array}{l}\text { CAP } \\
\text { (immunocompromised patients [100\%]) }\end{array}$ & $\begin{array}{l}\text { NPPV: } 48 \\
(14) \\
\text { COT: } 50 \\
(12)\end{array}$ & $\begin{array}{l}\text { NPPV: } 141 \\
\text { (24) } \\
\text { COT: } 136(23)\end{array}$ \\
\hline $\begin{array}{l}\text { Levitt } \\
2001[41]\end{array}$ & 38 & NPPV & COT & 1. Intubation & $\mathrm{CHF}$ & $\begin{array}{l}\text { NPPV: } 67 \\
(15) \\
\text { COT: } 69 \\
(15)\end{array}$ & NA \\
\hline $\begin{array}{l}\text { Ferrer } \\
2003[54]\end{array}$ & 105 & NPPV & COT & $\begin{array}{l}\text { 1. Mortality (in-ICU, } 90 \\
\text { days) } \\
\text { 2. Intubation }\end{array}$ & $\begin{array}{l}\text { AHRF } \\
\text { (Pneumonia [32.4\%]) }\end{array}$ & $\begin{array}{l}\text { NPPV: } 61 \\
(17) \\
\text { COT: } 62 \\
(18)\end{array}$ & $\begin{array}{l}\text { NPPV: } 102 \\
(21) \\
\text { COT: } 103(23)\end{array}$ \\
\hline $\begin{array}{l}\text { L'Her } \\
2004[44]\end{array}$ & 89 & NPPV & COT & $\begin{array}{l}\text { 1. Mortality (48 h, in- } \\
\text { hospital) }\end{array}$ & $\begin{array}{l}\text { CPE } \\
\text { (respiratory tract infection [33.7\%]) }\end{array}$ & $\begin{array}{l}\text { NPPV: } 84 \\
(6) \\
\text { COT: } 84(6)\end{array}$ & $\begin{array}{l}\text { NPPV: } 157 \\
\text { (71) } \\
\text { COT: } 167(73)\end{array}$ \\
\hline $\begin{array}{l}\text { Park } \\
2004[38]\end{array}$ & 80 & NPPV & COT & $\begin{array}{l}\text { 1. Mortality (in-hospital, } 15 \\
\text { days, } 60 \text { days) } \\
\text { 2. Intubation }\end{array}$ & $\begin{array}{l}\text { CPE } \\
\text { (myocardial ischemia [37.5\%]) }\end{array}$ & $\begin{array}{l}\text { NPPV: } 64 \\
(15) \\
\text { COT: } 65 \\
(15)\end{array}$ & NA \\
\hline $\begin{array}{l}\text { Gray } \\
2008[53]\end{array}$ & 1069 & NPPV & COT & $\begin{array}{l}\text { 1. Mortality (7 days, } 30 \\
\text { days) } \\
\text { 2. Intubation }\end{array}$ & $\begin{array}{l}\text { CPE } \\
\text { (ischemic heart disease [17.6\%]) }\end{array}$ & $\begin{array}{l}\text { NPPV: } 77 \\
(10) \\
\text { COT: } 79 \text { (9) }\end{array}$ & NA \\
\hline $\begin{array}{l}\text { Cosentini } \\
2010[55]\end{array}$ & 47 & NPPV & COT & $\begin{array}{l}\text { 1. Mortality (in-hospital) } \\
\text { 2. Intubation }\end{array}$ & CAP [100\%] & $\begin{array}{l}\text { NPPV: } 65 \\
\text { (17) } \\
\text { COT: } 72 \\
\text { (13) }\end{array}$ & $\begin{array}{l}\text { NPPV: } 249 \\
\text { (25) } \\
\text { COT: } 246(20)\end{array}$ \\
\hline $\begin{array}{l}\text { Qingyuan } \\
2012[35]\end{array}$ & 40 & NPPV & COT & $\begin{array}{l}\text { 1.Moratlity (in-ICU, in- } \\
\text { hospital) } \\
\text { 2. Intubation }\end{array}$ & $\begin{array}{l}\text { ALI } \\
\text { (immunocompromised patients [30\%]) }\end{array}$ & $\begin{array}{l}\text { NPPV: } 44 \\
\text { (14) } \\
\text { COT: } 49 \\
(14)\end{array}$ & $\begin{array}{l}\text { NPPV: } 225 \\
(17) \\
\text { COT: } 234(27)\end{array}$ \\
\hline $\begin{array}{l}\text { Elena } \\
2013[37]\end{array}$ & 80 & NPPV & COT & 1. Intubation & $\begin{array}{l}\text { AHRF } \\
\text { (pneumonia [100\%]) }\end{array}$ & NA & NA \\
\hline $\begin{array}{l}\text { Brambilla } \\
2014[52]\end{array}$ & 81 & NPPV & COT & 1. Intubation & Pneumonia [100\%] & $\begin{array}{l}\text { NPPV: } 65 \\
\text { (16) } \\
\text { COT: } 70 \\
(16)\end{array}$ & $\begin{array}{l}\text { NPPV: } 134 \\
\text { (32) } \\
\text { COT: } 148(44)\end{array}$ \\
\hline $\begin{array}{l}\text { Azevedo } \\
2015[47]\end{array}$ & 30 & NPPV & HFNC & 1. Intubation & $\begin{array}{l}\text { AHRF } \\
\text { (CHF [43\%]) }\end{array}$ & NA & NA \\
\hline Frat & 313 & NPPV/HFNC & СOT & 1. Mortality (in-ICU, 90 & AHRF & NPPV: 61 & NPPV: 149 \\
\hline
\end{tabular}


Table 1 Study populations, protocols, and characteristics (Continued)

\begin{tabular}{|c|c|c|c|c|c|c|c|}
\hline \multirow{2}{*}{$\begin{array}{l}\text { Author, } \\
\text { year }\end{array}$} & \multirow{2}{*}{$\begin{array}{l}\text { Sample } \\
\text { size } \\
n\end{array}$} & \multicolumn{3}{|l|}{ Protocols } & \multicolumn{3}{|l|}{ Baseline characteristics } \\
\hline & & $\begin{array}{l}\text { Intervention } \\
\text { setting }\end{array}$ & $\begin{array}{l}\text { Control } \\
\text { setting }\end{array}$ & Outcomes & $\begin{array}{l}\text { The main causes of acute respiratory } \\
\text { failure }\end{array}$ & Age, years & $\mathrm{PaO}_{2}: \mathrm{F}_{1} \mathrm{O}_{2}$ \\
\hline 2015 [9] & & & & $\begin{array}{l}\text { days) } \\
\text { 2. Intubation }\end{array}$ & (CAP [62.9\%]) & $\begin{array}{l}(17) \\
\text { HFNC: } 61 \\
(16) \\
\text { COT: } 59 \\
(17)\end{array}$ & $\begin{array}{l}(72) \\
\text { HFNC: } 157 \\
\text { (89) } \\
\text { COT: } 161 \text { (73) }\end{array}$ \\
\hline $\begin{array}{l}\text { Lemiale } \\
2015 \text { (1) [43] }\end{array}$ & 374 & NPPV & COT & $\begin{array}{l}\text { 1. Mortality ( } 28 \text { days) } \\
\text { 2. Intubation }\end{array}$ & $\begin{array}{l}\text { Pneumonia } \\
\text { (immunocompromised patients [100\%]) }\end{array}$ & $\begin{array}{l}\text { NPPV: } 61 \\
{[52-70]^{a}} \\
\text { COT: } 64 \\
{[53-72]^{a}}\end{array}$ & $\begin{array}{l}\text { NPPV: } 156 \\
{[95-248]^{\mathrm{a}}} \\
\text { COT: } 130 \\
{[86-205]^{\mathrm{a}}}\end{array}$ \\
\hline $\begin{array}{l}\text { Lemiale } \\
2015 \text { (2) [42] }\end{array}$ & 100 & HFNC & COT & 1. Intubation & $\begin{array}{l}\text { AHRF } \\
\text { (immunocompromised patients [100\%]) }\end{array}$ & $\begin{array}{l}\text { HFNC: } 59 \\
{[43-70]^{a}} \\
\text { COT: } 65 \\
{[53-72]^{a}}\end{array}$ & NA \\
\hline $\begin{array}{l}\text { Frat } \\
2016[58]\end{array}$ & 86 & NPPV/HFNC & COT & $\begin{array}{l}\text { 1. Mortality (in-ICU, } 90 \\
\text { days) } \\
\text { 2. Intubation }\end{array}$ & $\begin{array}{l}\text { AHRF } \\
\text { (immunocompromised patients [100\%]) }\end{array}$ & $\begin{array}{l}\text { Total: } 62 \\
{[48-74]}\end{array}$ & $\begin{array}{l}\text { Total: } 148 \\
(58)\end{array}$ \\
\hline $\begin{array}{l}\text { Jones } \\
2016[45]\end{array}$ & 303 & HFNC & COT & $\begin{array}{l}\text { 1. Mortality (in-hospital) } \\
\text { 2. Intubation }\end{array}$ & $\begin{array}{l}\text { AHRF } \\
\text { (COPD [23.9\%]) }\end{array}$ & $\begin{array}{l}\text { HFNC: } 75 \\
\text { (16) } \\
\text { COT: } 72 \\
(17)\end{array}$ & NA \\
\hline $\begin{array}{l}\text { Makdee } \\
2017[40]\end{array}$ & 128 & HFNC & COT & $\begin{array}{l}\text { 1. Mortality (7 days) } \\
\text { 2. Intubation }\end{array}$ & CPE & $\begin{array}{l}\text { HFNC: } 70 \\
(16) \\
\text { COT: } 71 \\
(14)\end{array}$ & NA \\
\hline $\begin{array}{l}\text { Azoulay } \\
2018[50]\end{array}$ & 778 & HFNC & COT & $\begin{array}{l}\text { 1. Mortality ( } 28 \text { days) } \\
\text { 2. Intubation }\end{array}$ & $\begin{array}{l}\text { AHRF } \\
\text { (immunocompromised patients [100\%]) }\end{array}$ & $\begin{array}{l}\text { HFNC: } 64 \\
{[55-70]^{a}} \\
\text { COT: } 63 \\
{[56-71]^{a}}\end{array}$ & $\begin{array}{l}\text { HFNC: } 136 \\
{[96-187]^{a}} \\
\text { COT: } 128 \\
{[92-164]^{a}}\end{array}$ \\
\hline $\begin{array}{l}\text { Doshi } \\
2018 \text { [57] }\end{array}$ & 228 & NPPV & HFNC & 1. Intubation & $\begin{array}{l}\text { AHRF } \\
\text { (COPD exacerbation [26.0\%]) }\end{array}$ & $\begin{array}{l}\text { NPPV: } 63 \\
(15) \\
\text { HFNC: } 63 \\
(14)\end{array}$ & NA \\
\hline $\begin{array}{l}\text { Eman } \\
2018[48]\end{array}$ & 70 & NPPV & HFNC & $\begin{array}{l}\text { 1. Mortality (in-hospital) } \\
\text { 2. Intubation }\end{array}$ & $\begin{array}{l}\text { AHRF } \\
\text { (interstitial lung disease [100\%]) }\end{array}$ & $\begin{array}{l}\text { NPPV: } 61 \\
(12) \\
\text { HFNC: } 61 \\
(12)\end{array}$ & $\begin{array}{l}\text { NPPV: } 166 \\
(42) \\
\text { HFNC: } 178 \\
(55)\end{array}$ \\
\hline $\begin{array}{l}\text { Hangyong } \\
2019[59]\end{array}$ & 200 & NPPV & COT & $\begin{array}{l}\text { 1. Mortality (in-hospital) } \\
\text { 2. Intubation }\end{array}$ & $\begin{array}{l}\text { AHRF } \\
\text { (pneumonia [100\%]) }\end{array}$ & $\begin{array}{l}\text { NPPV: } 53 \\
(18) \\
\text { COT: } 56 \\
(18)\end{array}$ & $\begin{array}{l}\text { NPPV: } 232 \\
\text { (35) } \\
\text { COT: } 231 \text { (28) }\end{array}$ \\
\hline $\begin{array}{l}\text { Andino } \\
2020[60]\end{array}$ & 46 & HFNC & СOT & $\begin{array}{l}\text { 1. Mortality (in-hospital) } \\
\text { 2. Intubation }\end{array}$ & $\begin{array}{l}\text { AHRF } \\
\text { (pneumonia [62\%]) }\end{array}$ & $\begin{array}{l}\text { HFNC: } 58 \\
\text { (19) } \\
\text { COT: } 61 \\
(11)\end{array}$ & $\begin{array}{l}\text { HFNC: } 96 \\
(29) \\
\text { COT: } 95 \text { (37) }\end{array}$ \\
\hline
\end{tabular}

AHRF Acute hypoxic respiratory failure; ALI Acute lung injury; ARDS, Acute respiratory distress syndrome; CAP Community-acquired pneumonia; CHF Congestive heart failure; COPD Chronic obstructive pulmonary disease; COT Conventional oxygen therapy; CPE Cardiogenic pulmonary edema; HFNC High-flow nasal cannula; ICU Intensive care unit; NPPV Noninvasive positive pressure ventilation

Continuous data were shown as mean and standard deviation, except for data labeled with "a".

${ }^{a}$ Data were reported as median and IQR (interquartile range).

hypoxic respiratory failure was pneumonia, followed by cardiopulmonary edema from CHF. Seven of the 27 included studies comprised many immunocompromised patients. The reported average $\mathrm{P} / \mathrm{F}$ at randomization differed among the studies (range 95-249).

\section{Risk of bias within studies}

Additional file 1 e-Fig 2 shows the risk of bias; although all studies did not blind their participants and clinicians to the intervention, the risk of bias in the other domains was low (e-Fig 2 in Additional file 1). Finally, all included studies were judged as having 
low risks of bias for outcomes (risk of bias across studies).

\section{Network meta-analysis}

The results of pairwise comparisons are shown in e-Fig 3 in Additional file 1 (short-term mortality) and e-Fig 4 in additional file 1 (Intubation). Additional file 1 e-Fig 5 shows the funnel plots of each outcome.

\section{Short-term mortality}

In the analysis of short-term mortality (including 20 studies), compared with COT, NPPV (RR, 0.88 [95\% CI, 0.76-1.01]; low certainty) and HFNC use (RR, 0.32 [95\% CI, 0.80-1.08]; low certainty) showed trends for lower mortality risk (Fig. 2a), and no significant difference was observed between NPPV and HFNC use for mortality (RR, 0.94 [95\% CI, 0.78-1.15]; low certainty). Anticipated absolute effects and 95\% CI between each of the two comparisons decreased by 28 per 1000 (95\% CI, 57 to +2$)$ in NPPV vs. COT, by 21 per 1000 (95\% CI, 61 to +24$)$ in HFNC use vs. COT, and by 9 per 1000 $(95 \% \mathrm{CI},-35$ to +24$)$ in NPPV vs. HFNC use (Table 2).

Confidence in the RR of each comparison and shortterm mortality assessed by the GRADE system is shown in Table 3. Incoherence between direct and indirect RRs was observed for all three comparisons determined by $p$ values of inconsistency. All comparisons (NPPV vs. COT, HFNC use vs. COT, and HFNC use vs. NPPV) showed "Major" concerns. The heterogeneity of all three comparisons resulted in "Major" concern outcomes due to the $95 \% \mathrm{CI}$ of the predicted risk ratio.

A ranking analysis revealed that the hierarchy for efficacy in reducing short-term mortality was HFNC use (SUCRA 72.1), followed by NPPV (SUCRA 68.8) and ultimately, COT (SUCRA 9.0) (Fig. 3a). The summary of findings of the NMA for short-term mortality is shown in Table 2. The estimate and certainty of the evidence of direct, indirect, and network comparisons are summarized in e-Table 3 in additional file 1.

\section{Endotracheal intubation}

Twenty-six studies were included in the analysis of endotracheal intubation. Compared with COT, NPPV (RR, 0.81 [95\% CI, 0.72-0.91]; low certainty) and HFNC use (RR, 0.78 [95\% CI, 0.68-0.89]; low certainty) were associated with statistically significant lower risks of endotracheal intubation (Fig. 2b), while no significant difference was observed between NPPV and HFNC use in the association with endotracheal intubation (RR, 1.04 [95\% CI, 0.88-1.22]; low certainty). Anticipated absolute effects (95\% CI) between each of the two comparisons decreased by 57 per 1000 (95\% CI, -83 to -27$)$ in

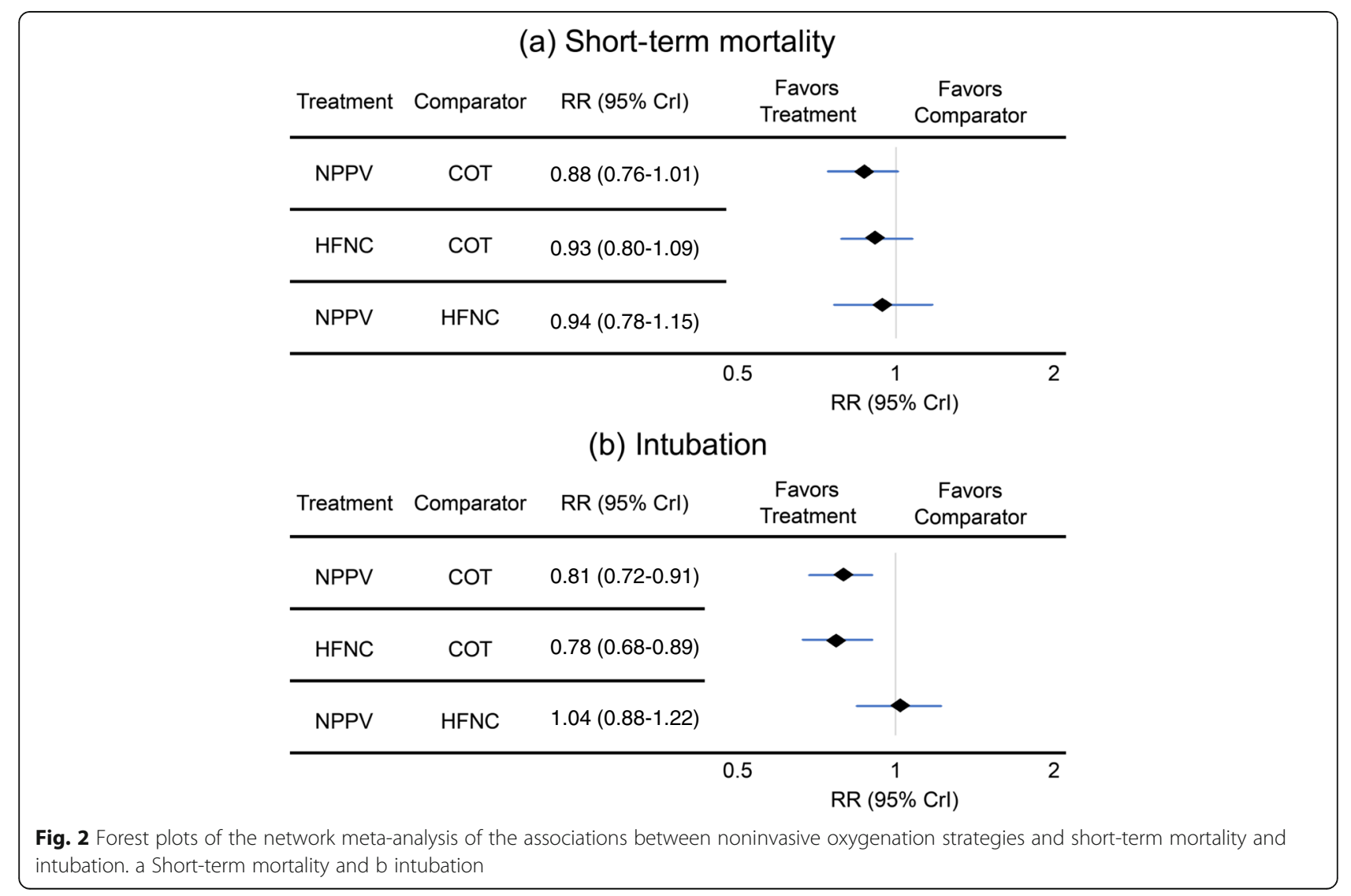


Table 2 Summary of findings of network meta-analysis for short-term mortality

\begin{tabular}{|c|c|c|c|c|c|c|c|c|}
\hline \multicolumn{9}{|c|}{ Estimates of effects, credible intervals, and certainly of the evidence for oxygen therapy in septic patients with acute respiratory failure. } \\
\hline \multicolumn{9}{|c|}{ BENEFITS } \\
\hline \multicolumn{6}{|c|}{$\begin{array}{l}\text { Patients or population: Septic patients with acute respiratory failure who need oxygen therapy } \\
\text { Interventions: One of the following oxygen therapies: NPPV, HFNT, or COT } \\
\text { Comparator (reference): One of the other therapies other than the therapy included in intervention } \\
\text { Outcome: Short-term mortality } \\
\text { Setting: In-hospital }\end{array}$} & $\overbrace{\substack{\text { Bob articiod } \\
\text { HFNC }}}$ & Network plot* & \\
\hline \multirow{2}{*}{\multicolumn{2}{|c|}{$\begin{array}{l}\text { Total studies: } 20 \\
\text { Total Patients: } 4,014\end{array}$}} & \multirow{3}{*}{ 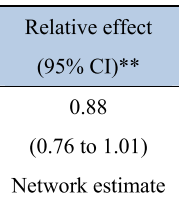 } & \multicolumn{3}{|c|}{ Anticipated absolute effect $(95 \% \mathrm{CI})^{* * *}$} & \multirow{2}{*}{$\begin{array}{l}\text { Certainly of the } \\
\text { evidence }\end{array}$} & \multirow{2}{*}{$\begin{array}{c}\text { Ranking**** } \\
\text { (SUCRA) }\end{array}$} & \multirow{2}{*}{$\begin{array}{c}\text { Interpretation of } \\
\text { Findings } \\
\end{array}$} \\
\hline & & & Without intervention & With intervention & Difference & & & \\
\hline O & $\begin{array}{l}\text { NPPV } \\
\text { (15 RCT; 2,559 } \\
\text { participants) } \\
\end{array}$ & & 237 per 1000 & 209 per 1000 & $\begin{array}{l}28 \text { fewer per } 1000 \\
\text { (57 fewer to } 2 \text { more) }\end{array}$ & $\begin{array}{c}\oplus \oplus \bigcirc \bigcirc \\
\text { Low }^{\mathrm{a}}\end{array}$ & $\begin{array}{c}1.6 \\
(68.8)\end{array}$ & $\begin{array}{l}\text { Probably } \\
\text { superior }\end{array}$ \\
\hline 0 & $\begin{array}{l}\text { HFNT } \\
\text { (6 RCT; 1,509 } \\
\text { participants) }\end{array}$ & $\begin{array}{c}0.93 \\
(0.80 \text { to } 1.08) \\
\text { Network estimate }\end{array}$ & 303 per 1000 & 282 per 1000 & $\begin{array}{c}21 \text { fewer per } 1000 \\
\text { (61 fewer to } 24 \text { more) }\end{array}$ & $\begin{array}{c}\oplus \oplus \bigcirc \bigcirc \\
\text { Low }^{\mathrm{a}}\end{array}$ & $\begin{array}{c}1.6 \\
(72.1)\end{array}$ & $\begin{array}{l}\text { Probably } \\
\text { superior }\end{array}$ \\
\hline O & COT & Reference & No estimable & No estimable & No estimable & Reference & 2.8 & Reference \\
\hline & & comparator & & & & comparator & $(9.0)$ & comparator \\
\hline & & & & & & & & \\
\hline & $\begin{array}{l}\text { NPPV } \\
\text { (3 RCT; } 338 \\
\text { participants) }\end{array}$ & $\begin{array}{c}0.94 \\
(0.78 \text { to } 1.15) \\
\text { Network estimate }\end{array}$ & 157 per 1000 & 148 per 1000 & $\begin{array}{l}9 \text { fewer per } 1000 \\
\text { ( } 35 \text { fewer to } 24 \text { more) }\end{array}$ & $\begin{array}{c}\oplus \oplus \bigcirc \bigcirc \\
\text { Low }^{\mathrm{a}}\end{array}$ & - & $\begin{array}{l}\text { Probably } \\
\text { equivalent }\end{array}$ \\
\hline & HFNC & $\begin{array}{l}\text { Reference } \\
\text { comparator }\end{array}$ & No estimable & No estimable & No estimable & $\begin{array}{l}\text { Reference } \\
\text { comparator }\end{array}$ & - & $\begin{array}{l}\text { Reference } \\
\text { comparator }\end{array}$ \\
\hline \multicolumn{9}{|c|}{$\begin{array}{l}\text { NMA, network meta-analysis: NPPV, non-invasive positive pressure ventilation; HFNC, high-flow nasal cannula; COT, conventional oxygen therapy; SOF, summary of findings: } \\
\text { SUCRA, surface under the cumulative ranking }\end{array}$} \\
\hline \multicolumn{9}{|c|}{ NMA-SoF table definitions } \\
\hline \multicolumn{9}{|c|}{${ }^{*}$ Lines represent direct comparisons } \\
\hline \multicolumn{9}{|c|}{ ** Estimates are reported as risk ratio. CI: confidence interval. } \\
\hline \multirow{2}{*}{\multicolumn{9}{|c|}{$\begin{array}{l}\text { *** Anticipated absolute effect. Anticipated absolute effect compares two risks by calculating the difference between the risks of the intervention group with the risk of the control } \\
\text { group. } \\
\text { **** Rank for efficacy outcome are presented. Rank statistics is defined as the probabilities that a treatment out of } n \text { treatments in a network meta-analysis is the best, the second, the } \\
\text { third and so on until the least effective treatment. }\end{array}$}} \\
\hline & & & & & & & & \\
\hline \multicolumn{9}{|c|}{ GRADE Working Group grades of evidence (or certainly in the evidence) } \\
\hline \multicolumn{9}{|c|}{ High quality: We are very confident that the true effect lies close to that of the estimate of the effect. } \\
\hline \multirow{3}{*}{\multicolumn{9}{|c|}{$\begin{array}{l}\text { Moderate quality: we are moderately confident in the effect estimate: The true effect is likely close to the estimate of the effect, but there is a possibility that it is substantially different } \\
\text { Low quality: Our confidence in the effect estimate is limited: The true effect may be substantially different from the estimate of the effect. } \\
\text { Very low quality: We have very little confidence in the effect estimate: The true effect is likely to be substantially different from the estimate effect }\end{array}$}} \\
\hline & & & & & & & & \\
\hline & & & & & & & & \\
\hline Expla & natory Footnotes & & & & & & & \\
\hline & value of inconsist & y was below 0.05 . & & & & & & \\
\hline
\end{tabular}

NPPV vs. COT and 70 per 1000 (95\% CI, - 101 to - 35) in HFNC use vs. COT, and increased by 9 per 1000 $(95 \%$ CI, -28 to +51$)$ in NPPV vs. HFNC use (Table 4).

Confidence in the RR of each comparison and intubation assessed according to the GRADE system (Table 3) showed incoherence between direct and indirect RRs for all three comparisons determined by the $p$ value of inconsistency. All comparisons (NPPV vs. COT, HFNC use vs. COT, and HFNC use vs. NPPV) showed "Major" concerns. The heterogeneity of all three comparisons resulted in "Major" concern due to the $95 \%$ CI of the predicted risk ratio. The ranking analysis revealed that the hierarchy for efficacy in reducing intubation was HFNC use (SUCRA 79.4), followed by NPPV (SUCRA 70.2), 
Table 3 Confidence in the relative risk of each comparison and outcome assessed by the GRADE system for short-term mortality and intubation

\begin{tabular}{|c|c|c|c|c|c|c|c|}
\hline & $\begin{array}{l}\text { Risk of bias across } \\
\text { studies }\end{array}$ & Imprecision & Heterogeneity & Indirectness & $\begin{array}{l}\text { Publication } \\
\text { bias }\end{array}$ & Incoherence & $\begin{array}{l}\text { Confidence in relative risk of } \\
\text { the event }\end{array}$ \\
\hline \multicolumn{8}{|c|}{ Short-term mortality } \\
\hline $\begin{array}{l}\text { NPPV vs. } \\
\text { COT }\end{array}$ & Undetected & $\begin{array}{l}\text { Not serious } \\
(95 \% \mathrm{Cl} 0.76- \\
1.01)\end{array}$ & $\begin{array}{l}\text { Major concern } \\
\text { a) } \\
(95 \% \text { PI 0.49- } \\
1.45)\end{array}$ & Low & $\begin{array}{l}\text { Not } \\
\text { suggested }\end{array}$ & $\begin{array}{l}\text { Major } \\
\text { concern }^{\mathrm{b}} \\
(p=0.005)\end{array}$ & $\begin{array}{l}\oplus \oplus \circ \circ \\
\text { Low }\end{array}$ \\
\hline $\begin{array}{l}\text { HFNC vs. } \\
\text { COT }\end{array}$ & Undetected & $\begin{array}{l}\text { Not serious } \\
(95 \% \text { Cl 0.80- } \\
1.08)\end{array}$ & $\begin{array}{l}\text { Major concern } \\
\text { a) } \\
(95 \% \text { PI 0.45- } \\
1.47)\end{array}$ & Low & $\begin{array}{l}\text { Not } \\
\text { suggested }\end{array}$ & $\begin{array}{l}\text { Major } \\
\text { concern }^{\mathrm{b}} \\
(p=0.031)\end{array}$ & $\begin{array}{l}\oplus \oplus \circ \circ \\
\text { Low }\end{array}$ \\
\hline $\begin{array}{l}\text { HFNC vs. } \\
\text { NPPV }\end{array}$ & Undetected & $\begin{array}{l}\text { Not serious } \\
(95 \% \text { Cl 0.78- } \\
1.15)\end{array}$ & $\begin{array}{l}\text { Major concern } \\
\text { a) } \\
(95 \% \text { PI 0.56- } \\
1.91)\end{array}$ & Low & $\begin{array}{l}\text { Not } \\
\text { suggested }\end{array}$ & $\begin{array}{l}\text { Major } \\
\text { concern }^{\mathrm{b}} \\
(p<0.001)\end{array}$ & $\begin{array}{l}\oplus \oplus \circ \circ \\
\text { LoW }\end{array}$ \\
\hline \multicolumn{8}{|l|}{ Intubation } \\
\hline $\begin{array}{l}\text { NPPV vs. } \\
\text { COT }\end{array}$ & Undetected & $\begin{array}{l}\text { Not serious } \\
(95 \% \text { Cl 0.72- } \\
0.91)\end{array}$ & $\begin{array}{l}\text { Major concern } \\
\text { a) } \\
(95 \% \text { PI 0.29- } \\
1.46)\end{array}$ & Low & $\begin{array}{l}\text { Not } \\
\text { suggested }\end{array}$ & $\begin{array}{l}\text { Major } \\
\text { concern }^{\mathrm{b}} \\
(p=0.0035)\end{array}$ & $\begin{array}{l}\oplus \oplus \circ \circ \\
\text { LoW }\end{array}$ \\
\hline $\begin{array}{l}\text { HFNC vs. } \\
\text { COT }\end{array}$ & Undetected & $\begin{array}{l}\text { Not serious } \\
(95 \% \text { Cl 0.68- } \\
0.89)\end{array}$ & $\begin{array}{l}\text { Major concern } \\
\text { a) } \\
(95 \% \text { PI 0.26- } \\
1.44)\end{array}$ & Low & $\begin{array}{l}\text { Not } \\
\text { suggested }\end{array}$ & $\begin{array}{l}\text { Major } \\
\text { concern }^{\mathrm{b}} \\
(p=0.001)\end{array}$ & $\begin{array}{l}\oplus \oplus 0 \circ \\
\text { Low }\end{array}$ \\
\hline $\begin{array}{l}\text { HFNC vs. } \\
\text { NPPV }\end{array}$ & Undetected & $\begin{array}{l}\text { Not serious } \\
(95 \% \mathrm{Cl} 0.88- \\
1.22)\end{array}$ & $\begin{array}{l}\text { Major concern } \\
\text { a) } \\
(95 \% \text { PI 0.45- } \\
2.49)\end{array}$ & Low & $\begin{array}{l}\text { Not } \\
\text { suggested }\end{array}$ & $\begin{array}{l}\text { Major } \\
\text { concern }^{\mathrm{b}} \\
(p<0.001)\end{array}$ & $\begin{array}{l}\oplus \oplus 0 \circ \\
\text { Low }\end{array}$ \\
\hline
\end{tabular}

CI Confidence interval; COT Conventional oxygen therapy; HFNC High-flow nasal therapy; NPPV noninvasive positive pressure ventilation; PI Prediction interval a Prediction interval extends into clinically important effects in both directions.

${ }^{b} P$ value of inconsistency was below 0.05 .

and ultimately, COT (SUCRA 0.4) (Fig. 3b). Table 4 summarizes the NMA findings for intubation; e-Table 3 in additional file 1 summarizes the estimate and certainty of the evidence of direct, indirect, and network comparisons.

\section{Results of additional analyses}

Of the 27 RCTs included in this study, only 14 focused on a single cause: 5, immunocompromised status; 4, pneumonia; 3 , CHF; 1 , post-transplant solid tumors; and 1 , interstitial pneumonia. Therefore, the sensitivity analyses performed included the subgroup analysis of the severity of respiratory failure $(\mathrm{P} / \mathrm{F}<200)$ and the cause of respiratory failure (immunocompromised patients, excluding CHF/acute exacerbation of COPD patients). In all subgroup analyses, both short-term mortality and intubation rates were similar to those in the main analysis (e-Table 4 in Additional file 1).

\section{Discussion}

This systematic review and NMA showed that NPPV and HFNC use were associated with lower risks of intubation compared with COT rather than an improved mortality risk. The SUCRA values of intubation for
HFNC use and NPPV in the NMA showed similar effects to those for NPPV and HFNC use. These results are consistent with those of previous systematic reviews (non-NMA) [18, 21, 61]. A systematic review by Zhao et al. [21] that included 11 studies $(n=3459)$ compared HFNC use with COT or NPPV and found that unlike NPPV, HFNC use reduced the intubation rate compared with COT. Those studies differ from this study as they included many post-extubation studies. Despite the results of the previous review being consistent with those of our study-that HFNC use reduces the intubation rate compared with $\mathrm{COT}$-the inclusion of studies examining the prevention of re-intubation after extubation would result in a deviation from the clinical question in this study. The sample size was inadequate due to the lack of RCTs that directly compared NPPV and HFNC use, and previous reviews have not shown a significant difference between NPPV and HFNC use. To complement the limitations of the existing studies, a systematic review using an NMA was necessary.

This NMA is the second study to demonstrate the effectiveness of NPPV and HFNC use in ARF. Ferreyro et al. [62] first reported an NMA describing the effects of noninvasive oxygenation strategies (e.g., NPPV and 


\section{(a) Short-term mortality}

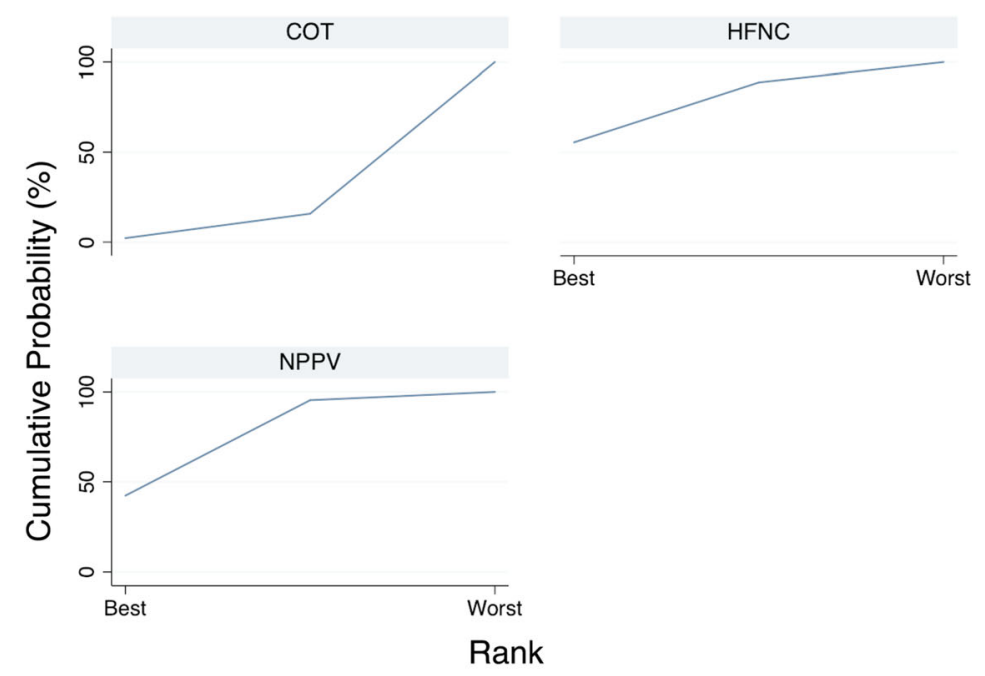

(b) Intubation

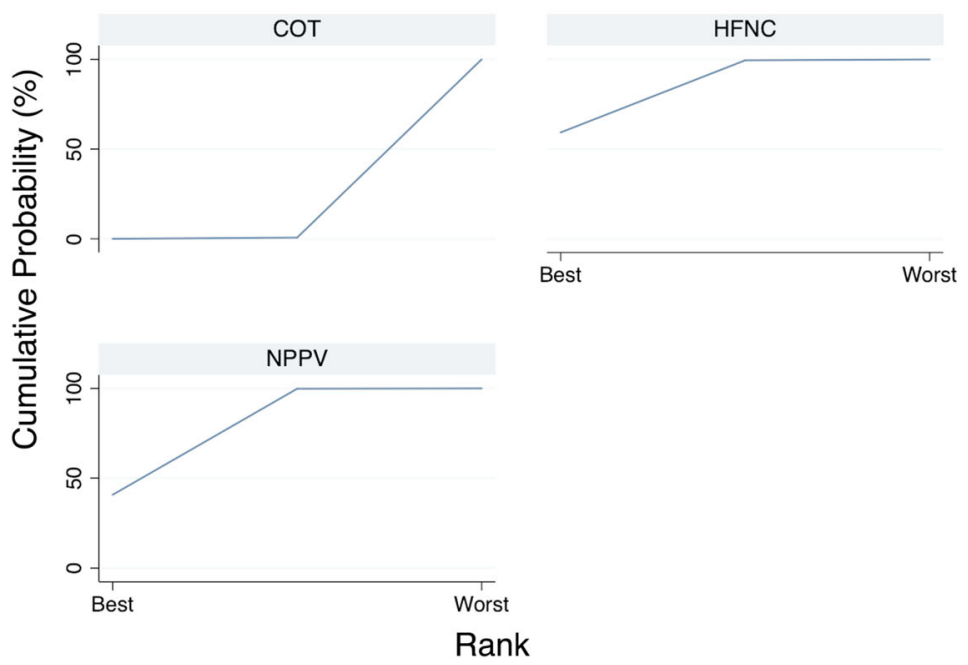

Fig. 3 Surface under the cumulative ranking of each noninvasive oxygen strategies for short-term mortality and intubation: a short-term mortality, $\mathbf{b}$ intubation

HFNC use) for patients with acute hypoxic respiratory failure. They concluded that treatment with noninvasive respiratory support devices was associated with a low mortality risk compared with standard oxygen therapy. Although the results of the intubation rate in this NMA are similar to those in the NMA by Ferreyro et al., the effect of NPPV and HFNC use on mortality, compared with that of COT, differed from the results in Ferreyro et al.'s study. In this NMA, we found no significant differences in the mortality risk between NPPV or HFNC use and COT.

Differences in the number of studies included in the NMA due to differences in the study inclusion criteria may have influenced the differences in the results of the two NMAs. First, this NMA included a large proportion of patients with CHF. Ferreyro et al. excluded studies in which patients with CHF constituted the majority of the study population. The clinical presentations of the cases of pneumonia and CHF are often complicated, with pneumonia being reported as a precipitating factor in CHF. Therefore, excluding studies that had patients with CHF may have affected the results of Ferreyro et al.'s NMA. Second, they included studies wherein patients with COPD constituted $<50 \%$ of the population while excluding studies that had a majority (> 50\%) of patients with COPD. As noninvasive oxygenation strategies are useful in COPD [63], these studies with populations mostly consisting of patients with COPD may have influenced the NMA results. Additionally, the inclusion of patients with COPD increased the heterogeneity of the 
Table 4 Summary of findings of network meta-analysis for intubation

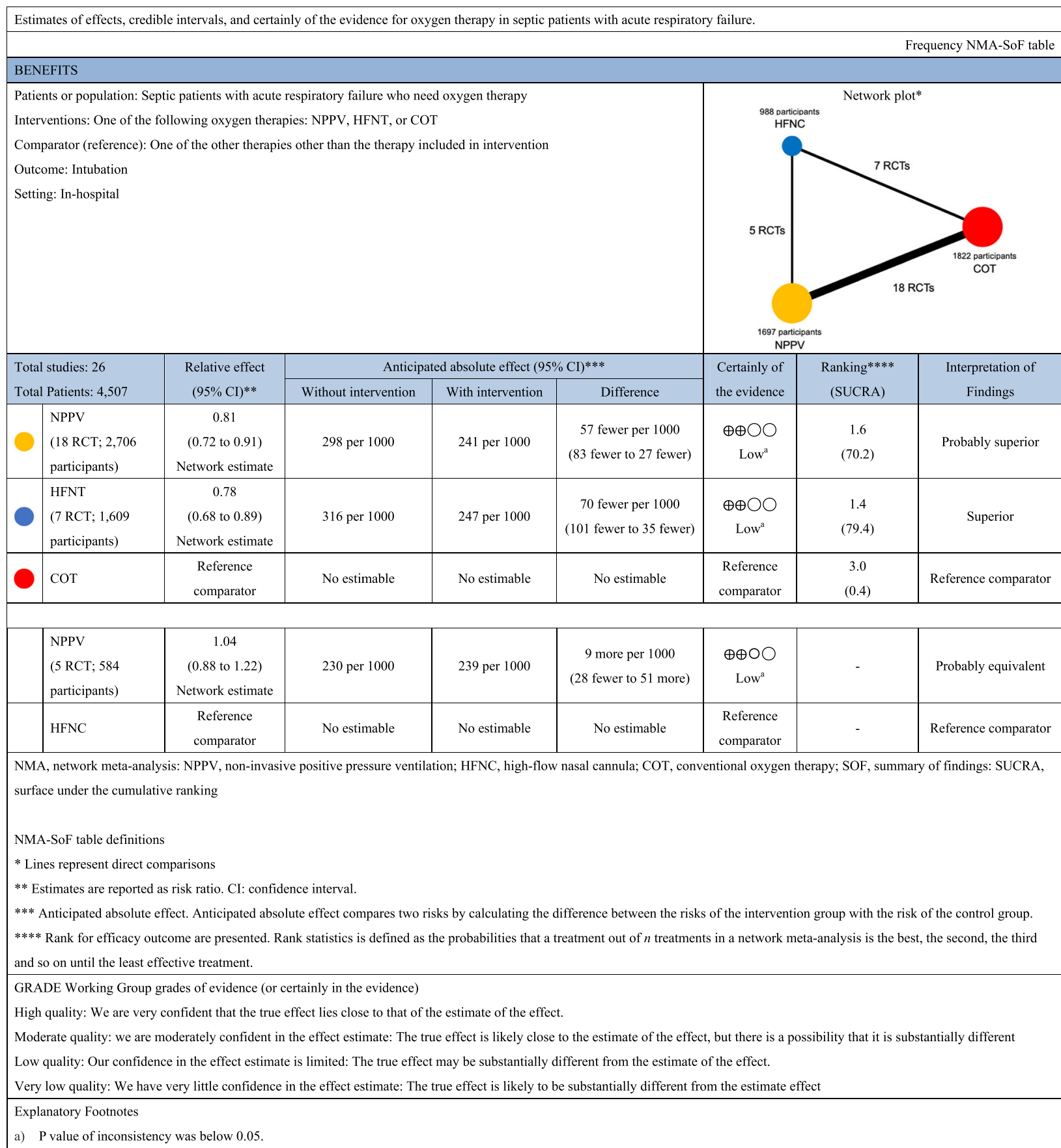

study population. Third, the NMA by Ferreyro et al. included the studies of ARF that occurred after abdominal surgery and chest trauma-associated respiratory failure, while excluding studies of patients after major cardiovascular surgery. Following abdominal surgery, diaphragmatic dysfunction and decreased vital lung capacity can cause atelectasis, resulting in hypoxemic respiratory failure (HRF). However, in pneumonia, which is the main cause of acute HRF, respiratory failure is caused by the decreased functional residual capacity due to inflammatory leachate in the alveoli and ventilator-perfusion mismatch. Analyzing these distinct pathogeneses of respiratory failure in a similar way is problematic and will affect the interpretation of results.

\section{Implications}

For patients, respiratory management without intubation is obviously more comfortable. Despite the superiority of 
HFNC use and NPPV being inconclusive in our study, noninvasive respiratory management is useful because per 1000 patients, utilizing NPPV and HFNC will help avoid intubation in 57-70 patients compared with COT. IMV is associated with various adverse events (e.g., VILI and VAP) and needs specific skills for comfortable management. Respiratory management that avoids tracheal intubation can reduce such complications, and the patient is relieved of the intubation discomfort. For the hospital manager, the costs of respiratory management may decrease due to the lower rate of intubation as daily ventilation costs increase healthcare costs by $59 \%$ compared with non-ventilation costs [64].

\section{Limitations}

This study had several limitations. First, there may be heterogeneity among the studies included, which may have affected the results. The NMA assumption is that the individual trials enrolled similar populations, and the intervention protocol was similar across different studies. Statistical heterogeneity is affected by a consequence of clinical or methodological diversity. Although statistical heterogeneity was a major concern in the mortality and intubation results, the clinical heterogeneity of diseases, including heart failure, pneumonia, and COPD, which were evaluated in this NMA, may not be considered high because of the difficulty in distinguishing between those diseases in the early stages of real-world clinical practice. The studies included in this NMA could not be clearly categorized by the cause of acute hypoxic respiratory failure because they did not include patients with a single cause. Therefore, sensitivity and subgroup analyses could not be performed.

Second, all RCTs included had high risks of performance bias due to the dramatic differences between HFNC use, COT, and NPPV, which made blinding impossible.

Third, although the statistical analysis was based on the assumption that there was no effect modifier, the inclusion of patients with various degrees of respiratory failure and different outcomes may have influenced the results due to undetectable effect modifiers. However, even for outcomes with different baseline risks, the relative effects of the interventions can remain consistent [65]. A sensitivity analysis based on respiratory failure severity should have been conducted; however, it was not performed because of the possibly similar severity (e.g., $\mathrm{P} / \mathrm{F} \sim 200$ ) of the patients in most studies included. Lastly, a few studies compared NPPV and HFNC use, and thus, the sample size was insufficient to compare NPPV and HFNC use. However, a trend in the direction of a difference is present, which may be further evaluated in the future if more studies compare these two noninvasive oxygenation strategies.

\section{Conclusions}

The results of this NMA show that both NPPV and HFNC use are associated with lower risks of endotracheal intubation; however, no significant differences in short-term mortality exist between these respiratory support devices.

\section{Abbreviations \\ ARF: Acute respiratory failure; CENTRAL: Cochrane central register of controlled trials; Cl: Confidence interval; COPD: Chronic obstructive pulmonary disease; COT: Conventional oxygen therapy; HFNC: High-flow nasal cannula; HRF: Hypoxemic respiratory failure; ICU: Intensive care unit; IMV: Invasive mechanical ventilation; NMA: Network meta-analysis; NPPV: Noninvasive positive-pressure ventilation; P/F ratio: Fractional inspired oxygen; RCT: Randomized controlled trial; RevMan: Review manager; RR: Relative risk; SUCRA: Surface under the cumulative ranking curve; VAP: Ventilator-associated pneumonia; VILI: Ventilator-induced lung injury}

\section{Supplementary Information}

The online version contains supplementary material available at https://doi. org/10.1186/s40560-021-00539-7.

Additional file 1: e-Table 1. PRISMA NMA Checklist of Items to Include When Reporting A Systematic Review Involving a Network Meta-analysis. e-Table 2. Search strategy. e-Table 3. Estimate and certainty of the evidence of direct, indirect, and network comparison: (a) Short-term mortality. (b) Intubation. e-Table 4. Summary of sensitivity analysis for the association of all interventions with outcomes: (a) Short-term mortality. (b) Intubation. e-Fig 1. PRISMA flow diagram (search, inclusion, and exclusion). e-Fig 2. Risk of bias summary for each comparison: (a) NPPV vs. COT. (b) HFNC vs. COT. (c) HFNC vs. NPPV. COT: conventional oxygen therapy, HFNC: high-flow nasal cannula oxygen, NPPV: noninvasive positive pressure ventilation. e-Fig 3. Forest plots for the pairwise comparison of short-term mortality: (a) NPPV vs. COT. (b) HFNC vs. COT. (c) HFNS vs. NPPV. COT: conventional oxygen therapy, HFNC: high-flow nasal cannula oxygen, NPPV: noninvasive positive pressure ventilation. e-Fig 4. Forest plots for the pairwise comparison of intubation: (a) NPPV vs. COT. (b) HFNC vs. COT. (c) HFNS vs. NPPV. COT: conventional oxygen therapy, HFNC: high-flow nasal cannula oxygen, NPPV: noninvasive positive pressure ventilation. e-Fig 5. Funnel plot of the comparison for NPPV and COT in each outcome: (a) Short-term mortality. (b) Intubation. COT: conventional oxygen therapy, NPPV: noninvasive positive pressure ventilation

\section{Acknowledgements}

We thank Editage for proofreading this manuscript.

\section{Authors' contributions}

HY participated in designing the study, acquiring data, performing statistical analyses, interpreting the data, and drafting the manuscript. $\mathrm{HO}$ conceived the study and participated in the interpretation of data, acquisition of data, and drafting the manuscript. TM conceived the study, participated in the interpretation of data, acquisition of data, and drafted the manuscript. MN conceived the study, participated in the designing of the study and interpretation of data, and drafted the manuscript. NS conceived the study, participated in designing the study and interpreting the data, and drafted the manuscript. The authors read and approved the final manuscript.

\section{Funding}

Not applicable

\section{Availability of data and materials}

The datasets generated during and/or analyzed during the current study are not publicly available due to post hoc analyses by co-authors but are available from the corresponding author on reasonable request. 


\section{Declarations}

Ethics approval and consent to participate

Not applicable

\section{Consent for publication}

Not applicable

\section{Competing interests}

The authors declare that they have no competing interests.

\begin{abstract}
Author details
'Department of Emergency and Critical Care Medicine, Jichi Medical University Saitama Medical Center, 1-847, Amanuma-cho, Oomiya-ku, Saitama-shi, Saitama 330-8503, Japan. ${ }^{2}$ Department of Clinical Research Education and Training Unit, Keio University Hospital Clinical and Translational Research Center (CTR), 35, Shinanomachi, Shinjuku-ku, Tokyo 160-8582, Japan. ${ }^{3}$ Department of Critical and Emergency Medicine, National Hospital Organization Yokohama Medical Center, 2-60-3, Harajyuku, Totsuka-ku, Yokohama-shi, Kanagawa 245-8575, Japan. ${ }^{4}$ Department of Cardiovascular Medicine, Graduate School of Medical Science, Kanazawa University, 1-13, Takaramachi, Kanazawa-shi, Ishikawa 920-0934, Japan. ${ }^{5}$ Department of Emergency and Critical Care Medicine, Yamagata University Hospital, 2-2-2, lidanishi, Yamagata-shi, Yamagata 990-2331, Japan. ${ }^{6}$ Department of Emergency and Critical Care Medicine, Postgraduate Schoo of Medical Science, Hiroshima University Hospital, 3-2-1, Kasumi, Minami-ku, Hiroshima-shi, Hiroshima 734-8551, Japan.
\end{abstract}

\section{Received: 16 December 2020 Accepted: 21 February 2021}

\section{Published online: 12 April 2021}

\section{References}

1. Ware LB, Matthay MA. The acute respiratory distress syndrome. N Engl J Med. 2000;342:1334-49.

2. Cook D, Brower R, Cooper J, Brochard L, Vincent JL. Multicenter clinical research in adult critical care. Crit Care Med. 2002;30:1636-43.

3. Schettino G, Altobelli N, Kacmarek RM. Noninvasive positive-pressure ventilation in acute respiratory failure outside clinical trials: experience at the Massachusetts General Hospital. Crit Care Med. 2008;36:441-7.

4. Esteban A, Anzueto A, Frutos F, Alía I, Brochard L, Stewart TE, Benito S, Epstein SK, Apezteguía C, Nightingale P, Arroliga AC, Tobin MJ, Mechanical Ventilation International Study Group. Characteristics and outcomes in adult patients receiving mechanical ventilation: a 28-day international study. JAMA. 2002:287:345-55.

5. Kollef MH. What is ventilator-associated pneumonia and why is it important? Respir Care. 2005;50:714-21.

6. Boles JM, Bion J, Connors A, Herridge M, Marsh B, Melot C, Pearl R, Silverman $\mathrm{H}$, Stanchina M, Vieillard-Baron A, Welte T. Weaning from mechanical ventilation. Eur Respir J. 2007;29:1033-56.

7. Demoule A, Girou E, Richard JC, Taille S, Brochard L. Increased use of noninvasive ventilation in French intensive care units. Intensive Care Med. 2006;32:1747-55.

8. Thille AW, Richard JC, Brochard L. The decision to extubate in the intensive care unit. Am J Respir Crit Care Med. 2013;187:1294-302.

9. Frat JP, Thille AW, Mercat A, Girault C, Ragot S, Perbet S, Prat G, Boulain T, Morawiec E, Cottereau A, Devaquet J, Nseir S, Razazi K, Mira JP, Argaud L, Chakarian JC, Ricard JD, Wittebole X, Chevalier S, Herbland A, Fartoukh M, Constantin JM, Tonnelier JM, Pierrot M, Mathonnet A, Béduneau G, Delétage-Métreau C, Richard JCM, Brochard L, Robert R, FLORALI Study Group, Network REVA. High-flow oxygen through nasal cannula in acute hypoxemic respiratory failure. N Engl J Med. 2015;372:2185-96.

10. Hernández G, Vaquero C, González P, Subira C, Frutos-Vivar F, Rialp G, Laborda C, Colinas L, Cuena R, Fernández R. Effect of postextubation highflow nasal cannula vs conventional oxygen therapy on reintubation in lowrisk patients: a randomized clinical trial. JAMA. 2016;315:1354-61.

11. Zhang Y, Fang C, Dong BR, Wu T, Deng JL. Oxygen therapy for pneumonia in adults. Cochrane Database Syst Rev. 2012;3:CD006607.

12. Xu XP, Zhang XC, Hu SL, Xu JY, Xie JF, Liu SQ, Liu L, Huang YZ, Guo FM, Yang Y, Qiu HB. Noninvasive ventilation in acute hypoxemic nonhypercapnic respiratory failure: a systematic review and meta-analysis. Crit Care Med. 2017:45:e727-33.
13. Bello G, De Pascale G, Antonelli M. Noninvasive ventilation. Clin Chest Med. 2016:37:711-21.

14. Hill NS. Complications of noninvasive ventilation. Respir Care. 2000;45:480-1.

15. Rochwerg B, Granton D, Wang DX, Helviz Y, Einav S, Frat JP, MekontsoDessap A, Schreiber A, Azoulay E, Mercat A, Demoule A, Lemiale V, Pesenti A, Riviello ED, Mauri T, Mancebo J, Brochard L, Burns K. High flow nasal cannula compared with conventional oxygen therapy for acute hypoxemic respiratory failure: a systematic review and meta-analysis. Intensive Care Med. 2019;45:563-72.

16. Xu Z, Li Y, Zhou J, Li X, Huang Y, Liu X, Burns KEA, Zhong N, Zhang H. Highflow nasal cannula in adults with acute respiratory failure and after extubation: a systematic review and meta-analysis. Respir Res. 2018;19:202.

17. Monro-Somerville T, Sim M, Ruddy J, Vilas M, Gillies MA. The effect of high-flow nasal cannula oxygen therapy on mortality and intubation rate in acute respiratory failure: a systematic review and meta-analysis. Crit Care Med. 2017:45:e449-56.

18. Ni YN, Luo J, Yu H, Liu D, Liang BM, Yao R, Liang ZA. Can high-flow nasal cannula reduce the rate of reintubation in adult patients after extubation? a meta-analysis. BMC Pulm Med. 2017;17:142.

19. AlYami MA, AlAhmari MD, Alotaibi H, AlRabeeah S, AlBalawi I, Mubasher M. Evaluation of efficacy of non-invasive ventilation in non-COPD and nontrauma patients with acute hypoxemic respiratory failure: a systematic review and meta-analysis. Ann Thorac Med. 2015;10:16-24.

20. Zhu $Y$, Yin $H$, Zhang $R$, Wei J. High-flow nasal cannula oxygen therapy versus conventional oxygen therapy in patients with acute respiratory failure: a systematic review and meta-analysis of randomized controlled trials. BMC Pulm Med. 2017;17:201

21. Zhao H, Wang H, Sun F, Lyu S, An Y. High-flow nasal cannula oxygen therapy is superior to conventional oxygen therapy but not to noninvasive mechanical ventilation on intubation rate: a systematic review and metaanalysis. Crit Care. 2017;21:184

22. Ni YN, Luo J, Yu H, Liu D, Liang BM, Liang ZA. The effect of high-flow nasal cannula in reducing the mortality and the rate of endotracheal intubation when used before mechanical ventilation compared with conventional oxygen therapy and noninvasive positive pressure ventilation. A systematic review and meta-analysis. Am J Emerg Med. 2018;36:226-33.

23. Hutton B, Salanti G, Caldwell DM, Chaimani A, Schmid CH, Cameron C, loannidis JPA, Straus S, Thorlund K, Jansen JP, Mulrow C, Catalá-López F, Gøtzsche PC, Dickersin K, Boutron I, Altman DG, Moher D. The PRISMA extension statement for reporting of systematic reviews incorporating network meta-analyses of health care interventions: checklist and explanations. Ann Intern Med. 2015;162:777-84.

24. TC C. Data collection form-intervention review for RCTs only. Secondary data collection form-intervention review for RCTs only. 2014. https://dplp. cochrane.org/data-extraction-forms (accessed September 8, 2019).

25. TC C. RevMan 5 Download and installation. Secondary RevMan 5 download and installation. 2014. https://community.cochrane.org/help/tools-and-softwa re/revman-5/revman-5-download/installation (accessed September 8, 2019).

26. TC C. Cochrane handbook for systematic reviews of interventions. 2011. https://training.cochrane.org/handbook (accessed September 8, 2019).

27. TC C. Table 8.5.d: Criteria for judging risk of bias in the 'Risk of bias' assessment tool. Secondary Table 8.5.d: Criteria for judging risk of bias in the 'Risk of bias' assessment tool. 2011. http://handbook-5-1.cochrane.org (accessed September 8, 2019).

28. Higgins JP, Thompson SG. Quantifying heterogeneity in a meta-analysis. Stat Med. 2002;21:1539-58.

29. Sterne JA, Sutton AJ, loannidis JP, Terrin N, Jones DR, Lau J, Carpenter J, Rücker G, Harbord RM, Schmid CH, Tetzlaff J, Deeks JJ, Peters J, Macaskill P, Schwarzer G, Duval S, Altman DG, Moher D, Higgins JPT. Recommendations for examining and interpreting funnel plot asymmetry in meta-analyses of randomised controlled trials. BMJ. 2011;343:d4002.

30. Puhan MA, Schunemann HJ, Murad MH, Li T, Brignardello-Petersen R, Singh JA, Kessels AG, Guyatt GH, Group GW. A GRADE Working Group approach for rating the quality of treatment effect estimates from network metaanalysis. BMJ. 2014;349:95630.

31. Yepes-Nunez JJ, Li SA, Guyatt G, Jack SM, Brozek JL, Beyene J, Murad MH, Rochwerg B, Mbuagbaw L, Zhang Y, Florez ID, Siemieniuk RA, Sadeghirad B, Mustafa R, Santesso N, Schunemann HJ. Development of the summary of findings table for network meta-analysis. J Clin Epidemiol. 2019;115:1-13.

32. Salanti G, Ades AE, loannidis JP. Graphical methods and numerical summaries for presenting results from multiple-treatment meta-analysis: an overview and tutorial. J Clin Epidemiol. 2011;64:163-71. 
33. Rhodes KM, Turner RM, Higgins JP. Predictive distributions were developed for the extent of heterogeneity in meta-analyses of continuous outcome data. J Clin Epidemiol. 2015;68:52-60.

34. Higgins JP, Jackson D, Barrett JK, Lu G, Ades AE, White IR. Consistency and inconsistency in network meta-analysis: concepts and models for multi-arm studies. Res Synth Methods. 2012;3:98-110.

35. Zhan Q, Sun B, Liang L, Yan X, Zhang L, Yang J, Wang L, Ma Z, Shi L, Wei L, Li G, Yang L, Shi Z, Chen Y, Xu Q, Li W, Zhu X, Wang Z, Sun Y, Zhuo J, Liu Y, Li X, Wang C. Early use of noninvasive positive pressure ventilation for acute lung injury: a multicenter randomized controlled trial. Crit Care Med. 2012; 40:455-60.

36. Wysocki M, Tric L, Wolff MA, Millet $H$, Herman B. Noninvasive pressure support ventilation in patients with acute respiratory failure. A randomized comparison with conventional therapy. Chest. 1995;107:761-8.

37. Prina E, Brambilla AM, Aliberti S, Piffer F, Bozzano V, Visintin B, Cellini A, Forno MD, Corradi F, Ferrari G, Nava S, Pelosi P, Cosentini R. Non-invasive continuous positive airway pressure versus oxygen Venturi in severe acute respiratory failure due to pneumonia: a randomized controlled trial. European Respiratory Society Annual Congress. 2013;42:P4764.

38. Park M, Sangean MC, Volpe Mde S, Feltrim MIZ, Nozawa E, Leite PF, Amato MBP, Lorenzi-Filho G. Randomized, prospective trial of oxygen, continuous positive airway pressure, and bilevel positive airway pressure by face mask in acute cardiogenic pulmonary edema. Crit Care Med. 2004;32:2407-15.

39. Masip J, Betbesé AJ, Páez J, Vecilla F, Cañizares R, Padró J, Paz MA, de Otero J, Ballús J. Non-invasive pressure support ventilation versus conventional oxygen therapy in acute cardiogenic pulmonary oedema: a randomised trial. Lancet. 2000;356:2126-32.

40. Makdee O, Monsomboon A, Surabenjawong U, Praphruetkit N, Chaisirin W, Chakorn T, Permpikul C, Thiravit P, Nakornchai T. High-flow nasal cannula versus conventional oxygen therapy in emergency department patients with cardiogenic pulmonary edema: a randomized controlled trial. Ann Emerg Med. 2017;70:465-72.

41. Levitt MA. A prospective, randomized trial of BiPAP in severe acute congestive heart failure. J Emerg Med. 2001;21:363-9.

42. Lemiale V, Mokart D, Mayaux J, Lambert J, Rabbat A, Demoule A, Azoulay E. The effects of a 2 -h trial of high-flow oxygen by nasal cannula versus Venturi mask in immunocompromised patients with hypoxemic acute respiratory failure: a multicenter randomized trial. Crit Care. 2015;19:380.

43. Lemiale $V$, Mokart $D$, Resche-Rigon M, Pène F, Mayaux J, Faucher E, Nyunga $M$, Girault C, Perez P, Guitton C, Ekpe K, Kouatchet A, Théodose I, Benoit D, Canet E, Barbier F, Rabbat A, Bruneel F, Vincent F, Klouche K, Loay K, Mariotte E, Bouadma L, Moreau AS, Seguin A, Meert AP, Reignier J, Papazian L, Mehzari I, Cohen Y, Schenck M, Hamidfar R, Darmon M, Demoule A, Chevret S, Azoulay E, Groupe de Recherche en Réanimation Respiratoire du patient d'OncoHématologie (GRRR-OH). Effect of noninvasive ventilation vs oxygen therapy on mortality among immunocompromised patients with acute respiratory failure: a randomized clinical trial. JAMA. 2015;314:1711-179.

44. L'Her E, Duquesne F, Girou E, de Rosiere XD, Conte PL, Renault S, Allamy JP, Boles JM. Noninvasive continuous positive airway pressure in elderly cardiogenic pulmonary edema patients. Intensive Care Med. 2004;30:882-8.

45. Jones PG, Kamona S, Doran O, Sawtell F, Wilsher M. Randomized controlled trial of humidified high-flow nasal oxygen for acute respiratory distress in the emergency department: the hot-er study. Respir Care. 2016;61:291-9.

46. Hilbert G, Gruson D, Vargas F, Valentino R, Gbikpi-Benissan G, Dupon M, Reiffers J, Cardinaud JP. Noninvasive ventilation in immunosuppressed patients with pulmonary infiltrates, fever, and acute respiratory failure. $\mathrm{N}$ Engl J Med. 2001;344:481-7.

47. Azevedo JR, Montenegro WS, Leitao AL, Silva MM, Prazeres JS, Maranhao JP. High flow nasal cannula oxygen (hfnc) versus non-invasive positive pressure ventilation (nippv) in acute hypoxemic respiratory failure. A pilot randomized controlled trial. Intensive Care Med. 2015;3:1-2.

48. Shebl E, Embarak S. High-flow nasal oxygen therapy versus noninvasive ventilation in chronic interstitial lung disease patients with acute respiratory failure. Egypt J Chest Dis Tuberc. 2018:67:270.

49. Antonelli M, Conti G, Bufi M, Costa MG, Lappa A, Rocco M, Gasparetto A, Meduri GU. Noninvasive ventilation for treatment of acute respiratory failure in patients undergoing solid organ transplantation: a randomized trial. JAMA. 2000;283:235-41.

50. Azoulay E, Lemiale V, Mokart D, Nseir S, Argaud L, Pène F, Kontar L, Bruneel F, Klouche K, Barbier F, Reignier J, Berrahil-Meksen L, Louis G, Constantin JM, Mayaux J, Wallet F, Kouatchet A, Peigne V, Théodose I, Perez P, Girault C,
Jaber S, Oziel J, Nyunga M, Terzi N, Bouadma L, Lebert C, Lautrette A, Bigé N, Raphalen JH, Papazian L, Darmon M, Chevret S, Demoule A. Effect of high-flow nasal oxygen vs standard oxygen on 28-day mortality in immunocompromised patients with acute respiratory failure: the high randomized clinical trial. JAMA. 2018;320:2099-107.

51. Bersten AD, Holt AW, Vedig AE, Skowronski GA, Baggoley CJ. Treatment of severe cardiogenic pulmonary edema with continuous positive airway pressure delivered by face mask. N Engl J Med. 1991;325:1825-30.

52. Brambilla AM, Aliberti S, Prina E, Nicoli F, Forno MD, Nava S, Ferrari G, Corradi F, Pelosi P, Bignamini A, Tarsia P, Cosentini R. Helmet CPAP vs. oxygen therapy in severe hypoxemic respiratory failure due to pneumonia. Intensive Care Med. 2014;40:942-9.

53. Gray A, Goodacre S, Newby DE, Masson M, Sampson F, Nicholl J, Trialists CPO. Noninvasive ventilation in acute cardiogenic pulmonary edema. $\mathrm{N}$ Engl J Med. 2008;359:142-51.

54. Ferrer M, Esquinas A, Leon M, Gonzalez G, Alarcon A, Torres A. Noninvasive ventilation in severe hypoxemic respiratory failure: a randomized clinical trial. Am J Respir Crit Care Med. 2003;168:1438-44.

55. Cosentini R, Brambilla AM, Aliberti S, Bignamini A, Nava S, Maffei A, Martinotti R, Tarsia P, Monzani V, Pelosi P. Helmet continuous positive airway pressure vs oxygen therapy to improve oxygenation in communityacquired pneumonia: a randomized, controlled trial. Chest. 2010;138:114-20.

56. Delclaux C, L'Her E, Alberti C, Mancebo J, Abroug F, Conti G, Guérin C, Schortgen F, Lefort Y, Antonelli M, Lepage E, Lemaire F, Brochard L. Treatment of acute hypoxemic nonhypercapnic respiratory insufficiency with continuous positive airway pressure delivered by a face mask: a randomized controlled trial. JAMA. 2000;284:2352-60.

57. Doshi P, Whittle JS, Bublewicz M, Kearney J, Ashe T, Graham R, Salazar S, Ellis TW Jr, Maynard D, Dennis R, Tillotson A, Hill M, Granado M, Gordon N, Dunlap C, Spivey S, Miller TL. High-velocity nasal insufflation in the treatment of respiratory failure: a randomized clinical trial. Ann Emerg Med. 2018;72:73-83.

58. Frat JP, Ragot S, Girault C, Perbet S, Prat G, Boulain T, Demoule A, Ricard JD, Coudroy R, Robert R, Mercat A, Brochard L, Thille AW, REVA network. Effect of non-invasive oxygenation strategies in immunocompromised patients with severe acute respiratory failure: a post-hoc analysis of a randomised trial. Lancet Respir Med 2016; 4: 646-652.

59. He H, Sun B, Liang L, Li Y, Wang H, Wei L, Li G, Guo S, Duan J, Li Y, Zhou Y, Chen $Y$, Li H, Yang J, Xu X, Song L, Chen J, Bao Y, Chen F, Wang P, Ji L, Zhang $Y$, Ding $Y$, Chen L, Wang $Y$, Yang L, Yang T, Weng H, Li H, Wang D, Tong J, Sun Y, Li R, Jin F, Li C, He B, Sun L, Wang C, Hu M, Yang X, Luo Q, Zhang J, Tan H, Wang C, Group ES. A multicenter RCT of noninvasive ventilation in pneumonia-induced early mild acute respiratory distress syndrome. Crit Care. 2019;23:300.

60. Andino R, Vega G, Pacheco SK, Arevalillo N, Leal A, Fernandez L, Rodriguez MJ. High-flow nasal oxygen reduces endotracheal intubation: a randomized clinical trial. Ther Adv Respir Dis. 2020;14:1753466620956459.

61. Luo J, Wang MY, Zhu H, Liang BM, Liu D, Peng XY, Wang RC, Li CT, He CY, Liang ZA. Can non-invasive positive pressure ventilation prevent endotracheal intubation in acute lung injury/acute respiratory distress syndrome? A meta-analysis. Respirology. 2014;19:1149-57.

62. Ferreyro BL, Angriman F, Munshi L, Sorbo LD, Ferguson ND, Rochwerg B, Ryu MJ, Saskin R, Wunsch H, da Costa BR, Scales DC. Association of noninvasive oxygenation strategies with all-cause mortality in adults with acute hypoxemic respiratory failure: a systematic review and meta-analysis. JAMA. 2020;324:57-67.

63. Osadnik CR, Tee VS, Carson-Chahhoud KV, Picot J, Wedzicha JA, Smith BJ. Non-invasive ventilation for the management of acute hypercapnic respiratory failure due to exacerbation of chronic obstructive pulmonary disease. Cochrane Database Syst Rev. 2017;7:Cd004104.

64. Kaier K, Heister T, Wolff J, Wolkewitz M. Mechanical ventilation and the daily cost of ICU care. BMC Health Serv Res. 2020;20:267.

65. Jansen JP, Fleurence R, Devine B, Itzler R, Barrett A, Hawkins N, Lee K, Boersma C, Annemans L, Cappelleri JC. Interpreting indirect treatment comparisons and network meta-analysis for health-care decision making: report of the ISPOR task force on indirect treatment comparisons good research practices: Part 1. Value Health. 2011;14:417-28.

\section{Publisher's Note}

Springer Nature remains neutral with regard to jurisdictional claims in published maps and institutional affiliations. 